Generalized $A$-statistical convergence and a Korovkin type approximation theorem for double sequences

\author{
Cemal Belen and Mustafa Yildirim
}




\title{
GENERALIZED $A$-STATISTICAL CONVERGENCE AND A KOROVKIN TYPE APPROXIMATION THEOREM FOR DOUBLE SEQUENCES
}

\author{
CEMAL BELEN AND MUSTAFA YILDIRIM
}

Received February 21, 2012

\begin{abstract}
In this paper, we use the notion of ideal to obtain two more general concepts and call them as $I$ - $A$-statistical convergence and ideal $A$-summability. We also use the the concept of $I$ - $A$-statistical convergence to prove a Korovkin type approximation theorem for double sequences of positive linear operators and present an interesting example to show that our Korovkin theorem is stronger than those proved earlier.
\end{abstract}

2000 Mathematics Subject Classification: 40A35; 41A36

Keywords: ideal convergence, statistical convergence, $A$-summability, Korovkin theorem, double sequence

\section{INTRODUCTION}

In [12], Gadjiev and Orhan has given a more general form of the classical Korovkin theorem (see [14]) by means of statistical convergence. Later, using different summability methods, some Korovkin type theorems have been obtained by many authors in several ways. For instance, see [4], [6-9], [10]. In recent years, the studies on double sequences has a rapid growth. Some concepts related to the single sequences have been extended to double sequences. In this work, we first generalize the concepts of $A$-statistical convergence and statistical $A$-summability for double sequences via ideals, and we call them as $d$ - $A$-statistical convergence and ideal $A$ summability. The another main purpose of this paper is to obtain a Korovkin type approximation theorem through the concept of $d$ - $A$-statistical convergence.

We refer the readers to [16] and [3] for more details on statistical convergence and ideal convergence of double sequences.

The authors were supported by the Scientific Research Project Fund of Cumhuriyet University under the project number F334. 


\section{2. $S_{A}^{2}(d)$-CONVERGENCE AND $d^{2}(A)$-SUMMABILITY}

Let $A=\left(a_{j k}^{m n}\right), m, n, j, k \in \mathbb{N}$, be a four dimensional matrix and $x=\left(x_{j k}\right)$ be a double sequence. Then the double (transformed) sequence, $A x:=\left(y_{m n}\right)$, is denoted by

$$
y_{m n}:=\sum_{j=1}^{\infty} \sum_{k=1}^{\infty} a_{j k}^{m n} x_{j k}
$$

where it is assumed that the summation exists as a well-known Pringsheim convergence (or P-limit) for each $(m, n) \in \mathbb{N} \times \mathbb{N}$. Now, let $A=\left(a_{j k}^{m n}\right)$ be a nonnegative $R H$-regular matrix (see, [13],[17]), $x=\left(x_{j k}\right)$ be a double sequence and $\left(y_{m n}\right)$ be defined as in (2.1). If $\left(y_{m n}\right)$ is statistically convergent to $L$, then $x$ is said to be statistically $A$-summable to $L$ (see $[1,5]$ ). Note that the concept of statistical $A$ summability for single sequences is introduced by Edely and Mursaleen in [11].

In this section, we first generalize the concepts of $A$-statistical convergence and statistical $A$-summability to $d$ - $A$-statistical convergence and ideal $A$-summability for double sequences, when $A=\left(a_{j k}^{m n}\right)$ is a nonnegative $R H$-regular matrix and $d$ is an admissible ideal in $\mathbb{N} \times \mathbb{N}$. We further establish the relation between these more general forms of summability methods.

Definition 1. A double sequence $x=\left(x_{j k}\right)$ is said to be $d$ - $A$-statistically convergent (or briefly $S_{A}^{2}(\mathcal{d})$-convergent) to a number $L$ if for each $\varepsilon>0$ and $\delta>0$

$$
\left\{(m, n) \in \mathbb{N} \times \mathbb{N}: \sum_{(j, k):\left|x_{j k}-L\right| \geq \varepsilon} a_{j k}^{m n} \geq \delta\right\} \in \mathcal{d} .
$$

In this case we write $S_{A}^{2}(\mathcal{l})-\lim x=L$.

Note that $\ell$ - $A$-statistically convergence for single sequences has been recently introduced by Savaş et al. (see [18]).

Definition 2. A double sequence $x=\left(x_{j k}\right)$ is said to be ideal $A$-summable (or briefly $d^{2}(A)$-summable) to a number $L$ if for each $\delta>0$

$$
\left\{(m, n) \in \mathbb{N} \times \mathbb{N}:\left|y_{m n}-L\right| \geq \delta\right\} \in \mathcal{d} .
$$

In this case we write $d^{2}(A)-\lim x=L$.

Remark 1. (i) Let $d_{0}:=\{A \subset \mathbb{N} \times \mathbb{N}:(\exists m(A) \in \mathbb{N})(j, k \geq m(A) \Rightarrow(j, k) \notin A)\}$. If we take $d=d_{0}$ in Definition 1 , then $S_{A}^{2}(d)$-convergence is reduced to $A$-statistical convergence. Also for $A=C(1,1), S_{A}^{2}(\mathcal{l})$-convergence becomes $S_{2}(\mathcal{l})$-convergence introduced by Belen and Yildirim in [2].

(ii) Let $d_{\delta_{2}}=\left\{K \subset \mathbb{N} \times \mathbb{N}: \delta_{2}(K)=0\right\}$, where $\delta_{2}(K)$ is the double $A$-density of $K$. 
If we take $d=d_{\delta_{2}}$ and $A=C(1,1)$, which is the double Cesàro matrix, in Definition 2, then $d^{2}(A)$-summability coincides with statistical $C(1,1)$-summability introduced by Móricz in [15]. But the choice of $d=d_{\delta_{2}}$ in Definition 2 gives us the concept of statistical $A$-summability (see [1] and [5]).

Recall that a nontrivial ideal of $\mathbb{N} \times \mathbb{N}$ is called strongly admissible if $\{i\} \times \mathbb{N}$ and $\mathbb{N} \times\{i\}$ belong to $d$ for each $i \in \mathbb{N}$.

Theorem 1. Let $A=\left(a_{j k}^{m n}\right)$ be a nonnegative $R H$-regular matrix and $d$ be a strongly admissible ideal in $\mathrm{N} \times \mathbb{N}$. If a double sequence is bounded and $S_{A}^{2}(\mathcal{Q})$ convergent to $L$, then it is $d^{2}(A)$-summable to $L$ but not conversely.

Proof. Let $x=\left(x_{j k}\right)$ be bounded and $S_{A}^{2}(\mathcal{l})$-convergent to $L$. Write $M:=\sup _{j, k}\left|x_{j k}-L\right|$ and $K(\varepsilon):=\left\{(j, k), j \leq m, k \leq n:\left|x_{j k}-L\right| \geq \varepsilon\right\}$ for any $\varepsilon>0$. Then, we have

$$
\begin{aligned}
& \left|y_{m n}-L\right|= \\
& =\left|\sum_{j, k=1,1}^{\infty, \infty} a_{j k}^{m n}\left(x_{i j}-L\right)+L\left(\sum_{j, k=1,1}^{\infty, \infty} a_{j k}^{m n}-1\right)\right| \\
& \leq\left|\sum_{j, k=1,1}^{\infty, \infty} a_{j k}^{m n}\left(x_{j k}-L\right)\right|+|L|\left|\sum_{j, k=1,1}^{\infty, \infty} a_{j k}^{m n}-1\right| \\
& \leq\left|\sum_{(j, k) \in K(\varepsilon)} a_{j k}^{m n}\left(x_{j k}-L\right)\right|+\left|\sum_{(j, k) \notin K(\varepsilon)} a_{j k}^{m n}\left(x_{j k}-L\right)\right|+|L|\left|\sum_{j, k=1,1}^{\infty, \infty} a_{j k}^{m n}-1\right| \\
& \leq M \sum_{(j, k) \in K(\varepsilon)} a_{j k}^{m n}+\varepsilon \sum_{(j, k) \notin K(\varepsilon)} a_{j k}^{m n}+|L|\left|\sum_{j, k=1,1}^{\infty, \infty} a_{j k}^{m n}-1\right| \\
& \leq B\left\{\sum_{(j, k) \in K(\varepsilon)} a_{j k}^{m n}+\left|\sum_{j, k=1,1}^{\infty, \infty} a_{j k}^{m n}-1\right|\right\}+\varepsilon\|A\|
\end{aligned}
$$

where $\|A\|=\sup _{m, n} \sum_{j} \sum_{k}\left|a_{j k}^{m n}\right|<\infty$ and $B=\max (M,|L|)$. Now choose a $\delta>0$ such that $\delta-\varepsilon\|A\|>0$. Hence from the last inequality we obtain

$$
\begin{gathered}
\left\{(m, n) \in \mathbb{N} \times \mathbb{N}:\left|y_{m n}-L\right| \geq \delta\right\} \\
\subset\left\{n \in \mathbb{N}: \sum_{(j, k) \in K(\varepsilon)} a_{j k}^{m n} \geq \frac{\delta-\varepsilon\|A\|}{2 B}\right\} \cup\left\{n \in \mathbb{N}:\left|\sum_{j, k=1,1}^{\infty, \infty} a_{j k}^{m n}-1\right| \geq \frac{\delta-\varepsilon\|A\|}{2 B}\right\} \\
=: K_{1} \cup K_{2} .
\end{gathered}
$$


By using definition of $S_{A}^{2}(\mathcal{d})$-convergence we get $K_{1} \in \mathcal{d}$. Also since $d$ is strongly admissible and $A$ is $R H$-regular, we obtain that $d-\lim _{m, n}\left|\sum_{j, k} a_{j k}^{m n}-1\right|=0$, so $K_{2} \in d$. Hence, by definition of an ideal we conclude that $d^{2}(A)-\lim x=L$.

To show that the converse is not true in general, let $d$ be a strongly admissible ideal, $A=C(1,1)$ and $x=\left(x_{j k}\right)$ be defined as

$$
x_{j k}=(-1)^{j}, \text { for all } k .
$$

Since

$$
P-\lim _{m, n} \frac{1}{m n} \sum_{j=1}^{m} \sum_{k=1}^{n} x_{j k}=0,
$$

$x$ is $C(1,1)$-summable to zero (hence $d^{2}(C(1,1))$-summable to zero), but obviously $x$ is not $C(1,1)^{d}$-statistical convergent. This completes the proof of theorem.

\section{3. $S_{A}^{2}(\mathcal{Q})$-APPROXIMATION}

In this section we prove a Korovkin type approximation theorem via the concept of $S_{A}^{2}(\mathcal{l})$-convergence for a double sequence of positive linear operators defined on $C(K)$, where $C(K)$ is the space of all continuous real valued functions on any compact subset of the real two dimensional space. Note that $C(K)$ is a Banach space with the norm $\|\cdot\|_{C(K)}$ defined by

$$
\|f\|_{C(K)}:=\sup _{(x, y) \in K}|f(x, y)|,(f \in C(K)) .
$$

Let $L$ be a linear operator from $C(K)$ into $C(K)$. Then as usual, we say that $L$ is positive linear operator provided that $f \geq 0$ implies $L f \geq 0$. Also, we denote the value of $L f$ at a point $(x, y)$ by $L(f ; x, y)$. Before proceeding further, we quote here the classical and statistical forms of Korovkin-type theorems introduced in [20] and [6], respectively.

Theorem 2. Let $\left\{L_{j k}\right\}$ be a double sequence of positive linear operators acting from $C(K)$ into itself. Then for all $f \in C(K)$,

$$
P-\lim _{j, k}\left\|L_{j k}(f)-f\right\|_{C(K)}=0
$$

if and only if

$$
P-\lim _{j, k}\left\|L_{j k}\left(f_{i}\right)-f_{i}\right\|_{C(K)}=0 \quad \text { for } \quad i=0,1,2,3,
$$

where $f_{0}(x, y)=1, f_{1}(x, y)=x, f_{2}(x, y)=y, f_{3}(x, y)=x^{2}+y^{2}$. 
Theorem 3. Let $A=\left(a_{j k}^{m n}\right)$ be a nonnegative $R H$-regular matrix. Let $\left\{L_{j k}\right\}$ be a double sequence of positive linear operators acting from $C(K)$ into itself. Then for all $f \in C(K)$,

if and only if

$$
s t_{A}^{2}-\lim _{j, k}\left\|L_{j k}(f)-f\right\|_{C(K)}=0
$$

$$
s t_{A}^{2}-\lim _{j, k}\left\|L_{j k}\left(f_{i}\right)-f_{i}\right\|_{C(K)}=0 \quad \text { for } \quad i=0,1,2,3,
$$

where $f_{0}(x, y)=1, f_{1}(x, y)=x, f_{2}(x, y)=y, f_{3}(x, y)=x^{2}+y^{2}$.

Now we give the main result of this section.

Theorem 4. Let $\left\{L_{j k}\right\}$ be a double sequence of positive linear operators acting from $C(K)$ into itself. Then for all $f \in C(K)$,

$$
S_{A}^{2}(\mathcal{l})-\lim _{j, k}\left\|L_{j k}(f)-f\right\|_{C(K)}=0
$$

if and only if

$$
S_{A}^{2}(\mathcal{l})-\lim _{j, k}\left\|L_{j k}\left(f_{i}\right)-f_{i}\right\|_{C(K)}=0 \quad \text { for } i=0,1,2,3,
$$

where $f_{0}(x, y)=1, f_{1}(x, y)=x, f_{2}(x, y)=y, f_{3}(x, y)=x^{2}+y^{2}$.

Proof. Condition (3.2) follows immediately from condition (3.1) since each $f_{i} \in$ $C(K),(i=0,1,2,3)$. Let us prove the converse. By the continuity of $f$ on compact set $K$, we can write $|f(x, y)| \leq M$, where $M=\|f\|_{C(K)}$. Also since $f \in C(K)$, for every $\varepsilon>0$, there is a number $\delta>0$ such that $|f(u, v)-f(x, y)|<\varepsilon$ for all $(u, v) \in K$ satisfying $|u-x|<\delta$ and $|v-y|<\delta$. Hence we get

$$
|f(u, v)-f(x, y)|<\varepsilon+\frac{2 M}{\delta^{2}}\left\{(u-x)^{2}+(v-y)^{2}\right\} .
$$

Since $\left\{L_{j k}\right\}$ is linear and positive, we obtain from (3.3) that

$$
\begin{aligned}
\mid & L_{j k}(f ; x, y)-f(x, y) \mid \\
\leq & L_{j k}(|f(u, v)-f(x, y)| ; x, y)+|f(x, y)|\left|L_{j k}\left(f_{0} ; x, y\right)-f_{0}(x, y)\right| \\
\leq & L_{j k}\left(\varepsilon+\frac{2 M}{\delta^{2}}\left((u-x)^{2}+(v-y)^{2}\right) ; x, y\right) \\
& +|f(x, y)|\left|L_{j k}\left(f_{0} ; x, y\right)-f_{0}(x, y)\right| \\
\leq & \varepsilon+(\varepsilon+M)\left|L_{j k}\left(f_{0} ; x, y\right)-f_{0}\right|+\frac{2 M}{\delta^{2}}\left|L_{j k}\left(f_{3} ; x, y\right)-f_{3}(x, y)\right| \\
& +\frac{4 M}{\delta^{2}}|x|\left|L_{j k}\left(f_{1} ; x, y\right)-f_{1}(x, y)\right|+\frac{4 M}{\delta^{2}}|y|\left|L_{j k}\left(f_{2} ; x, y\right)-f_{2}(x, y)\right| \\
& +\frac{2 M}{\delta^{2}}\left(x^{2}+y^{2}\right)\left|L_{j k}\left(f_{0} ; x, y\right)-f_{0}(x, y)\right|
\end{aligned}
$$




$$
\begin{aligned}
\leq & \varepsilon+\left(\varepsilon+M+\frac{2 M}{\delta^{2}}\left(C^{2}+D^{2}\right)\right)\left|L_{j k}\left(f_{0} ; x, y\right)-f_{0}(x, y)\right| \\
& +\frac{2 M}{\delta^{2}}\left|L_{j k}\left(f_{3} ; x, y\right)-f_{3}(x, y)\right|+\frac{4 M C}{\delta^{2}}\left|L_{j k}\left(f_{1} ; x, y\right)-f_{1}(x, y)\right| \\
& +\frac{4 M D}{\delta^{2}}\left|L_{j k}\left(f_{2} ; x, y\right)-f_{2}(x, y)\right|
\end{aligned}
$$

where $C:=\max |x|, D:=\max |y|$. Taking supremum over $(x, y) \in K$ we get

$$
\left\|L_{j k}(f)-f\right\|_{C(K)} \leq B \sum_{i=0}^{3}\left\|L_{j k}\left(f_{i}(u, v) ; x, y\right)-f_{i}(x, y)\right\|_{C(K)}
$$

where

$$
B:=\max \left\{\varepsilon+M+\frac{2 M}{\delta^{2}}\left(C^{2}+D^{2}\right), \frac{2 M}{\delta^{2}}, \frac{4 M C}{\delta^{2}}, \frac{4 M D}{\delta^{2}}\right\} .
$$

For any $\sigma>0$ define

$$
\begin{aligned}
D & =\left\{(j, k) \in \mathbb{N} \times \mathbb{N}:\left\|L_{j k}(f ; x, y)-f(x, y)\right\|_{C(K)} \geq \sigma\right\} \\
D_{i} & =\left\{(j, k) \in \mathbb{N} \times \mathbb{N}:\left\|L_{j k}\left(f_{i} ; x, y\right)-f_{i}(x, y)\right\|_{C(K)} \geq \frac{\sigma}{4 B}\right\}, i=0,1,2,3 .
\end{aligned}
$$

Then $D \subset \bigcup_{i=0}^{3} D_{i}$ and hence

$$
\left\{(m, n) \in \mathbb{N} \times \mathbb{N}: \sum_{(j, k) \in D} a_{j k}^{m n} \geq \eta\right\} \subset \bigcup_{i=0}^{3}\left\{(m, n) \in \mathbb{N} \times \mathbb{N}: \sum_{(j, k) \in D_{i}} a_{j k}^{m n} \geq \eta\right\}
$$

for any $\eta>0$. From this inclusion and (3.2), we immediately get (3.1). This completes the proof of theorem.

We remark that if we take $d=\ell_{0}$ in Theorem 4 , we obtain Theorem 3 .

Now, we will show that Theorem 4 is stronger than its classical and statistical forms.

Example 1. Let $d$ be a strongly admissible ideal and $A \in \mathcal{d}$ be infinite set. Further let $\lambda=\left(\lambda_{m}\right)$ and $\mu=\left(\mu_{n}\right)$ be two non-decreasing sequences of positive numbers tending to $\infty$ such that

$$
\lambda_{m+1} \leq \lambda_{m}+1, \lambda_{1}=1 \text { and } \mu_{n+1} \leq \mu_{n}+1, \mu_{1}=1 .
$$

For the intervals $J_{m}:=\left[m-\lambda_{m}+1, m\right]$ and $I_{n}:=\left[n-\mu_{n}+1, n\right]$, consider the four dimensional matrix $A=\left(a_{j k}^{m n}\right)$ and the double sequence $x=\left(x_{j k}\right)$ given by

$$
a_{j k}^{m n}= \begin{cases}\frac{1}{\lambda_{m} \mu_{n}} & ; \text { if } j \in J_{m} \text { and } k \in I_{n} \\ 0 & ; \text { otherwise. }\end{cases}
$$


and

$$
x_{j k}= \begin{cases}j k & ; \text { if } m-\left[\sqrt{\lambda_{m}}\right]+1 \leq j \leq m, n-\left[\sqrt{\mu_{n}}\right]+1 \leq k \leq n,(m, n) \notin A \\ j k & ; \text { if } m-\lambda_{m}+1 \leq j \leq m, n-\mu_{n}+1 \leq k \leq n,(m, n) \in A \\ 0 & ; \text { otherwise }\end{cases}
$$

respectively. In this case $S_{A}^{2}(\mathcal{l})$-convergence coincides with $S_{\lambda, \mu}(\mathcal{\ell})$-convergence introduced in [2]. Also $x$ is neither $P$-convergent nor $A$-statistically convergent but $S_{A}^{2}(d)-\lim x=0$ (see again [2]). Now consider the following Bernstein operators (see [19]) given by

$$
\left.B_{j k}(f ; x, y)=\sum_{p=0}^{j} \sum_{q=0}^{k} f\left(\frac{p}{j}, \frac{q}{k}\right)\left(\begin{array}{l}
j \\
p
\end{array}\right)\left(\begin{array}{l}
k \\
q
\end{array}\right)\right) x^{p}(1-x)^{j-p} y^{q}(1-y)^{k-q},
$$

where $0 \leq x, y \leq 1$ and $f \in C\left(I^{2}\right) ; K:=I^{2}=[0,1] \times[0,1]$. Let $L_{j k}: C\left(I^{2}\right) \rightarrow$ $C\left(I^{2}\right)$ be defined by

$$
L_{j k}(f ; x, y)=\left(1+x_{j k}\right) B_{j k}(f ; x, y)
$$

where $\left(x_{j k}\right)$ is defined as in (3.4). Then observe that

$$
\begin{aligned}
& L_{j k}\left(f_{0} ; x, y\right)=\left(1+x_{j k}\right) f_{0}(x, y) \\
& L_{j k}\left(f_{1} ; x, y\right)=\left(1+x_{j k}\right) f_{1}(x, y) \\
& L_{j k}\left(f_{2} ; x, y\right)=\left(1+x_{j k}\right) f_{2}(x, y) \\
& L_{j k}\left(f_{3} ; x, y\right)=\left(1+x_{j k}\right)\left(f_{3}(x, y)+\frac{x-x^{2}}{j}+\frac{y-y^{2}}{k}\right) .
\end{aligned}
$$

Since $d$ is strongly admissible ideal (in this case $P$-convergence implies $S_{A}^{2}(\mathcal{d}$ )convergence), we obtain

$$
S_{A}^{2}(\mathcal{l})-\lim _{j, k}\left\|L_{j k}\left(f_{i}\right)-f_{i}\right\|_{C(K)}=0
$$

for $i=0,1,2,3$. Thus $\left(L_{j k}\right)$ satisfies the condition (3.2) of Theorem 4. Hence we have

$$
S_{A}^{2}(\mathcal{d})-\lim _{j, k}\left\|L_{j k}(f)-f\right\|_{C(K)}=0,
$$

for all $f \in C(K)$. But, since $\left(x_{j k}\right)$ is not convergent in Pringsheims's sense and $A$ statistical sense, we see that Theorem 2 and Theorem 3 do not work for our operators defined by (3.5). Hence it is shown that our version is more general to deal with the situation when the operators $L_{j k}$ do not satisfy the conditions of Theorem 2 and Theorem 3.

Finally by using trigonemetric test functions, we give the statement of Theorem 4 for a double sequence of positive linear operators defined on $C^{*}\left(\mathbb{R}^{2}\right)$, the space of 
all $2 \pi$-periodic and real valued continuous functions on $\mathbb{R}^{2}$. This space is equipped with the supremum norm

$$
\|f\|_{C^{*}\left(\mathbb{R}^{2}\right)}:=\sup _{(x, y) \in \mathbb{R}^{2}}|f(x, y)|,\left(f \in C^{*}\left(\mathbb{R}^{2}\right)\right) .
$$

Then using the similar technique as in the proof of Theorem 4, one can also get the following result.

Theorem 5. Let $\left\{L_{j k}\right\}$ be a double sequence of positive linear operator acting from $C^{*}\left(\mathbb{R}^{2}\right)$ into itself. Then for all $f \in C^{*}\left(\mathbb{R}^{2}\right)$

$$
S_{A}^{2}(\mathcal{l})-\lim _{j, k}\left\|L_{j k}(f)-f\right\|_{C^{*}\left(\mathbb{R}^{2}\right)}=0
$$

if and only if

$$
S_{A}^{2}(\mathcal{l})-\lim _{j, k}\left\|L_{j k}\left(f_{i}\right)-f_{i}\right\|_{C^{*}\left(\mathbb{R}^{2}\right)}=0,
$$

where $f_{0}(x, y)=1, f_{1}(x, y)=\sin x, f_{2}(x, y)=\sin y, f_{3}(x, y)=\cos x, f_{4}(x, y)=$ $\cos y$.

If the ideal $d$ in Theorem 5 is replaced by $d_{0}$, we immediately get the following result proved in [4].

Corollary 1. Let $\left\{L_{j k}\right\}$ be a double sequence of positive linear operator acting from $C^{*}\left(\mathbb{R}^{2}\right)$ into itself. Then for all $f \in C^{*}\left(\mathbb{R}^{2}\right)$

$$
s t_{A}^{2}-\lim _{j, k}\left\|L_{j k}(f)-f\right\|_{C^{*}\left(\mathbb{R}^{2}\right)}=0
$$

if and only if

$$
s t_{A}^{2}-\lim _{j, k}\left\|L_{j k}\left(f_{i}\right)-f_{i}\right\|_{C^{*}\left(\mathbb{R}^{2}\right)}=0,
$$

where $f_{0}(x, y)=1, f_{1}(x, y)=\sin x, f_{2}(x, y)=\sin y, f_{3}(x, y)=\cos x, f_{4}(x, y)=$ $\cos y$.

\section{REFERENCES}

[1] C. Belen, M. Mursaleen, and M. Yildirim, "Statistical $A$-summability of double sequences and a Korovkin type approximation theorem," Bull. Korean Math. Soc., vol. 49, no. 4, pp. 851-861, 2012.

[2] C. Belen and M. Yildirim, "On generalized statistical convergence of double sequences via ideals," Ann. Univ. Ferrara Sez. VII Sci. Mat., vol. 58, no. 1, pp. 11-20, 2012.

[3] P. Das, P. Kostyrko, W. Wilczyński, and P. Malik, "I and $I^{*}$-convergence of double sequences," Math. Slovaca, vol. 58, no. 5, pp. 605-620, 2008.

[4] K. Demirci and F. Dirik, "Four-dimensional matrix transformation and rate of A-statistical convergence of periodic functions," Math. Comput. Modelling, vol. 52, no. 9-10, pp. 1858-1866, 2010.

[5] K. Demirci and S. Karakuş, "Korovkin-type approximation theorem for double sequences of positive linear operators via statistical $A$-summability," Result. Math., vol. 63, no. 1-2, pp. 1-13, 2013. 
[6] F. Dirik and K. Demirci, "Korovkin type approximation theorem for functions of two variables in statistical sense," Turk. J. Math., vol. 34, no. 1, pp. 73-84, 2010.

[7] O. Duman, "A Korovkin type approximation theorems via $i$-convergence," Czech. Math. J., vol. 57, no. 1, pp. 367-375, 2007.

[8] O. Duman, M. K. Khan, and C. Orhan, " $A$-statistical convergence of approximating operators," Math. Inequal. Appl., vol. 6, no. 4, pp. 689-699, 2003.

[9] O. Duman and C. Orhan, "Statistical approximation by positive linear operators," Stud. Math., vol. 161, no. 2, pp. 187-197, 2004.

[10] O. H. H. Edely, S. A. Mohiuddine, and A. K. Noman, "Korovkin type approximation theorems obtained through generalized statistical convergence," Appl. Math. Lett., vol. 23, no. 11, pp. 13821387,2010

[11] O. H. H. Edely and M. Mursaleen, "On statistical A-summability," Math. Comput. Modelling, vol. 49, no. 3-4, pp. 672-680, 2009.

[12] A. D. Gadjiev and C. Orhan, "Some approximation theorems via statistical convergence," Rocky Mt. J. Math., vol. 32, no. 1, pp. 129-138, 2002.

[13] H. J. Hamilton, "Transformations of multiple sequences," Duke Math. J., vol. 2, pp. 29-60, 1936.

[14] P. P. Korovkin, Linear Operators and Approximation Theory. Delhi: Hindustan Publ. Co., 1960.

[15] F. Móricz, "Tauberian theorems for double sequences that are statistically summable $(C, 1,1)$," $J$. Math. Anal. Appl., vol. 286, no. 1, pp. 340-350, 2003.

[16] Mursaleen and O. H. H. Edely, "Statistical convergence of double sequences," J. Math. Anal. Appl., vol. 288, no. 1, pp. 223-231, 2003.

[17] G. M. Robison, "Divergent double sequences and series," Transactions A. M. S., vol. 28, pp. 5073, 1926.

[18] E. Savas, P. Das, and S. Dutta, "A note on strong matrix summability via ideals," Appl. Math. Lett., vol. 25, no. 4, pp. 733-738, 2012.

[19] D. D. Stancu, "A method for obtaining polynomials of Bernstein type of two variables," Am. Math. Mon., vol. 70, pp. 269-264, 1963.

[20] V. I. Volkov, "On the convergence of sequences of linear positive operators in the space of continuous functions of two variables," Dokl. Akad. Nauk SSSR, vol. 115, pp. 17-19, 1957.

\section{Authors' addresses}

\section{Cemal Belen}

Ordu University, Department of Mathematics Education, Faculty of Education, 52200, Ordu, Turkey E-mail address: cbelen52@gmail.com

\section{Mustafa Yildirim}

Cumhuriyet University, Department of Mathematics, Faculty of Science, 58140 Sivas, Turkey

E-mail address: yildirimecumhuriyet.edu.tr 